\title{
Electronic Excitation and Dynamic Promotion of a Surface Reaction
}

\author{
D. N. Denzler, ${ }^{1}$ C. Frischkorn, ${ }^{2}$ C. Hess, ${ }^{1}$ M. Wolf,${ }^{2}$ and G. Ertl ${ }^{1}$ \\ ${ }^{1}$ Fritz-Haber-Institut der Max-Planck-Gesellschaft, Faradayweg 4-6, 14195 Berlin, Germany \\ ${ }^{2}$ Fachbereich Physik, Freie Universität Berlin, Arnimallee 14, 14195 Berlin, Germany
}

(Received 17 April 2003; published 26 November 2003)

\begin{abstract}
The mechanism of recombinative desorption of hydrogen from a $\mathrm{Ru}(0001)$ surface induced by femtosecond-laser excitation has been investigated and compared to thermally initiated desorption. For the laser-driven process, it is shown that hot substrate electrons mediate the reaction within a few hundred femtoseconds resulting in a huge isotope effect between $\mathrm{H}_{2}$ and $\mathrm{D}_{2}$ in the desorption yield. In mixed saturation coverages, this ratio crucially depends on the proportions of $\mathrm{H}$ and $\mathrm{D}$. Deviations from second order desorption kinetics demonstrate that the recombination is dynamically promoted by excitation of neighboring, but nonreacting adatoms. A concentration dependent rate constant which accounts for the faster excitation of $\mathrm{H}$ versus $\mathrm{D}$ is proposed.
\end{abstract}

DOI: 10.1103/PhysRevLett.91.226102

Chemical reactions involving species adsorbed on a metal surface are mediated through excitation of electrons and/or phonons of the substrate. Since thermal equilibration between these excitations occurs on a femtosecond (fs) to picosecond (ps) time scale, the rate normally may be described to a very good approximation within the framework of transition state theory [1] in terms of the temperature dependent rate constant and as a function of the surface concentrations. Rapid absorption of a fs-laser pulse by the conduction electrons of the substrate may, however, trigger the onset of a surface reaction before equilibration between the heat baths of electrons and phonons is reached, as has been exemplified in the reaction between $\mathrm{O}$ and $\mathrm{CO}$ adsorbed on a $\mathrm{Ru}(0001)$ surface yielding the release of $\mathrm{CO}_{2}$ into the gas phase [2]. For adsorption on thin metal films with Schottky contact, it was recently demonstrated that nonadiabatic coupling to electron-hole pairs plays an important role in surface reactions and is not negligible even in low-energy processes in which phononic excitations are thought to dominate [3]. Hot electrons were proposed to routinely participate in substrate-mediated reactions contrary to the traditional picture of a thermal surface reaction, in which phonons solely drive the system over the reaction barrier in the electronic ground state.

Coadsorbed species on a metal substrate can modify the electronic structure and hence influence the surface reactivity. Altering the height of the reaction barrier in the electronic ground state and/or energetic shifts of the potential energy surface in electronically excited states are typical consequences. In the case of catalytic promotion (e.g., by alkali atoms), these static changes in the electronic potential energy landscape result in an enhanced reaction rate [4]. In this Letter, we report on dynamic promotion of a prototype surface reaction, $\mathrm{H}_{\mathrm{ad}}+$ $\mathrm{H}_{\mathrm{ad}} \rightarrow \mathrm{H}_{2 \text {,gas }}$ on $\mathrm{Ru}(0001)$. Hydrogen recombination may be initiated thermally (i.e., under conditions of thermal equilibrium between all degrees of freedom), but if in-
PACS numbers: $82.65 .+\mathrm{r}, 68.43 . \mathrm{Mn}, 78.90 .+\mathrm{t}, 82.53 .-\mathrm{k}$

duced by fs-laser excitation characteristic differences are observed: (i) The hydrogen molecules coming off the surface exhibit appreciable excess kinetic energy, (ii) there exists a large isotope effect between $\mathrm{H}$ and $\mathrm{D}$ (which is absent in the thermal reaction), and (iii) most remarkably, desorption of the heavier isotope $\left(D_{2}\right)$ is facilitated by the presence of the lighter counterpart $\left(\mathrm{H}_{\mathrm{ad}}\right)$ on the surface. This dynamic effect is attributed to the faster excitation of the lighter isotope. Its thereby initiated lateral displacement influences the energetics of the neighboring adspecies and thus promotes the desorption kinetics. In a more general sense, rate constants for a surface reaction $A+B \stackrel{R}{\rightarrow} A B$ may be reformulated as $k=$ $k\left(C^{*}\right)$ with $C^{*}$ being an excited, but not-reacting, coadsorbate as the results of this Letter demonstrate.

Hydrogen adsorbs dissociatively on $\mathrm{Ru}(0001)$ up to saturation $[\mathrm{H}-(1 \times 1)]$ with occupation of threefold fcc sites [5]. In the present experiments, the Ru sample is mounted in an ultrahigh vacuum chamber (base pressure $<1 \times 10^{-10} \mathrm{mbar}$ ) and can be temperature-controlled from 30 to 1530 K. Saturation of a pure H or D coverage is achieved by exposing the clean sample surface at $170 \mathrm{~K}$ to $\sim 50$ Langmuir $\mathrm{H}_{2}$ or $\mathrm{D}_{2}$, respectively [6]. Statistically mixed H:D coverages are obtained by first precovering the clean Ru surface with HD and subsequently saturating it with either of the pure isotopes. Thermal desorption is induced by resistively heating the sample (rate $1.5 \mathrm{~K} / \mathrm{s}$ ), while laser desorption is caused by fs pulses $(800 \mathrm{~nm}$, $130 \mathrm{fs}, 2.2 \mathrm{~mJ}, \sim 60 \mathrm{~J} / \mathrm{m}^{2}$ absorbed yield-weighted fluence [7]). The desorbing molecules are detected by a quadrupole mass spectrometer, whereby the collection efficiency is greatly enhanced by a glass enclosure around the ionization region [8]. Details of the experimental setup can be found elsewhere [7].

Typical thermal desorption spectroscopy (TDS) data for $\mathrm{H}_{2}$ and $\mathrm{D}_{2}$ are reproduced in Fig. 1(a). Two characteristic maxima around 320 and $380 \mathrm{~K}$ are in accordance with previous reports $[6,9]$ and are practically identical 


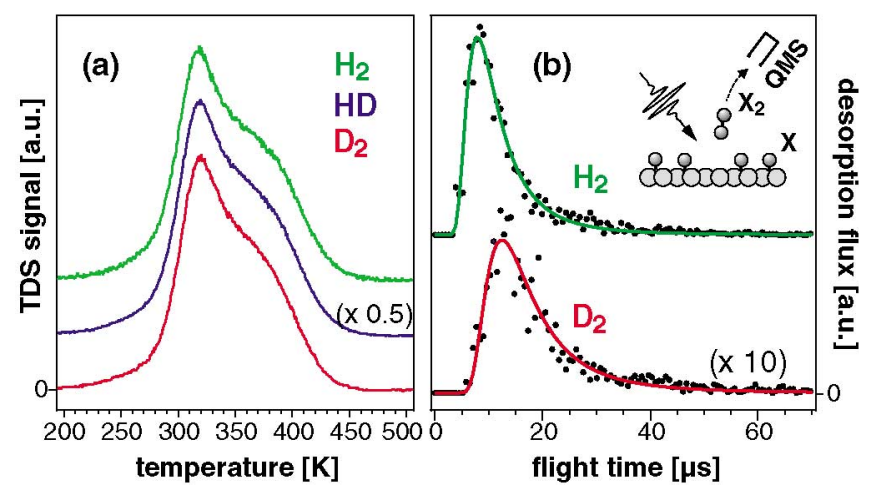

FIG. 1 (color). (a) TD spectra for $\mathrm{H}_{2}, \mathrm{HD}$, and $\mathrm{D}_{2}$ from a saturation coverage of HD. Spectra are scaled according to detection sensitivity and offset for clarity. (b) The fs-laser induced TOF spectra for $\mathrm{H}_{2}$ (offset) and $\mathrm{D}_{2}$. Mean kinetic energies are obtained by fitting modified Maxwell-Boltzmann distributions (solid lines) to the experimental data. $E_{\text {kin }}=(2110 \pm 400) \mathrm{K}$ and $(1720 \pm 290) \mathrm{K}$ for $\mathrm{H}_{2}$ and $\mathrm{D}_{2}$, respectively.

for both $\mathrm{H}_{2}$ and $\mathrm{D}_{2}$. This indicates that there is no noticeable isotope effect in the thermal desorption kinetics, presumably because the zero-point energy differences are very similar in the initial and final states. Recombinative desorption initiated by absorption of a fs-laser pulse exhibits quite different characteristics: From the time-of-flight (TOF) spectrum as shown in Fig. 1(b), a mean translational energy of about $2000 \mathrm{~K}$ is derived for the desorbing $\mathrm{H}_{2}$ and $\mathrm{D}_{2}$ molecules, i.e., a much higher value than one would obtain from TDS. This indicates that after fs-laser excitation the hydrogen molecules leave the surface excited without complete equilibration with the heat bath of the solid.

Closer insight into the dynamics of this reaction pathway is obtained by two-pulse correlation (2PC) experiments [10]. Two equally intense fs-laser pulses are sent onto the Ru sample with variable time delay $\Delta t$. In Fig. 2, the resulting desorption yields for $\mathrm{H}_{2}$ and $\mathrm{D}_{2}$ from $\mathrm{H}-(1 \times$ $1)$ and $\mathrm{D}-(1 \times 1)$ surfaces, respectively, are plotted as a function of $\Delta t$. The narrow full width at half maximum (FWHM) of $\sim 1 \mathrm{ps}$ is a clear indication for the operation of the hot-electron mechanism, since only for pulse separations shorter than the electron-phonon equilibration time ( $\sim 1 \mathrm{ps}$ for $\mathrm{Ru})$ the electron temperature is greatly enhanced due to the combined effect of both pulses. This in turn gives rise to a sharp peak in the $2 \mathrm{PC}$ of the $\mathrm{H}_{2}$ and $\mathrm{D}_{2}$ yield. In contrast, a phonon-mediated process proceeds on a much slower time scale of tens of picoseconds [2].

For theoretical modeling, the two-temperature model [13] in conjunction with the modified electronic friction model [11] accounting for the electron-mediated energy transfer from the $\mathrm{Ru}$ substrate to the $\mathrm{H} / \mathrm{D}$ adsorbate is employed. In this model, the coupling time $\tau_{\text {el }}$ between substrate and adsorbate is the inverse of the friction coefficient and scales proportionally with the mass of the

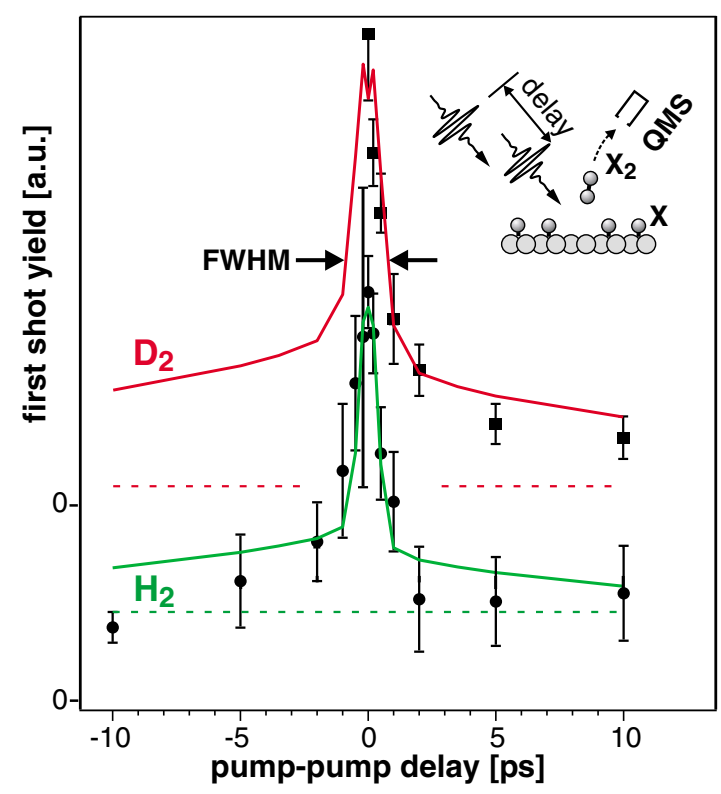

FIG. 2 (color). Two-pulse correlation (pulse intensity ratio 54:46) of the laser-induced desorption yield of hydrogen from $\mathrm{Ru}(0001)$. The yield of the first shot out of a series of laser pulses is shown as a function of the pulse-pulse delay $\left(D_{2}\right.$ data offset). Dashed lines mark the overall yield of both individual pulses infinitely separated in time. Solid lines are obtained using the electronic friction model of Ref. [11] with a FWHM of 1.1 and 1.5 ps for $\mathrm{H}_{2}$ and $\mathrm{D}_{2}$, respectively. The dip at very short delays originates from the competition between the heat transport into the bulk and electron-phonon coupling [12].

adsorbate. The solid lines in Fig. 2 fit the experimental data with a modified activation energy [14] of $1.35 \mathrm{eV}$ and $\tau_{\text {el }}=180 \mathrm{fs}$ and $360 \mathrm{fs}$ for $\mathrm{H}_{2}$ and $\mathrm{D}_{2}$, respectively. As illustrated in Fig. 3(a) for an exemplary time delay of 1 ps, absorption of the first fs-light pulse causes a rapid rise of the electron temperature $T_{\mathrm{el}}$ which couples within less than $1 \mathrm{ps}$ to the phonon temperature $T_{\mathrm{ph}}$. If the second pulse is absorbed after $\Delta t$, the yield of both desorbing molecules $\mathrm{H}_{2}$ and $\mathrm{D}_{2}$ will be enhanced if full equilibration of $T_{\mathrm{el}}$ and $T_{\mathrm{ph}}$ has not yet been reached, underlining the observed narrow FWHM of the 2PC experiments.

The second striking difference of the laser-driven process to thermal desorption concerns the isotope effect: If the yields per laser shot from pure $\mathrm{H}$ and $\mathrm{D}$ adlayers, respectively, are compared with each other, a ratio of about $10( \pm 2): 1$ results [see scaling factor in Fig. 1(b)]. Unlike in phonon-mediated processes [15], distinct isotope effects in the reaction yield are characteristic for processes involving electronic excitation, e.g., in electron stimulated desorption [16] as well as in surface femtochemistry [2], and have to be attributed to the mass-dependent distance the system travels on the electronically excited state during its short lifetime [17]. The principle underlying this process is illustrated by Fig. 3(b). Absorption of light by the conduction electrons creates hot electrons above the Fermi level $E_{\mathrm{F}}$. They rapidly equilibrate internally to an electron temperature $T_{\mathrm{el}}$ 

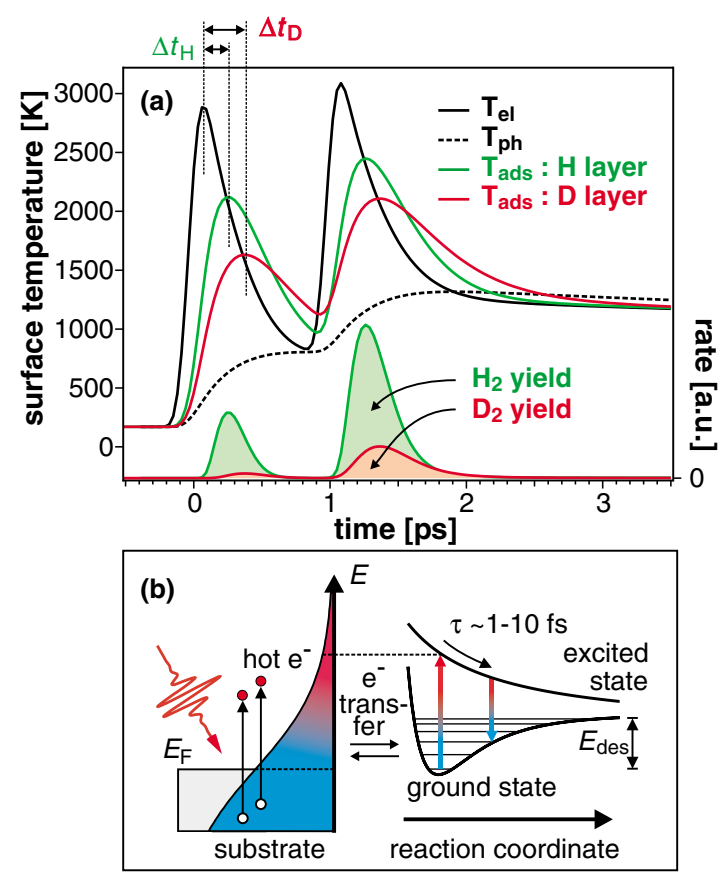

FIG. 3 (color). Energy transfer from substrate to adsorbate: (a) Based on the two-temperature model [13], the calculated time profiles of the electron and the phonon temperature $T_{\mathrm{el}}$ and $T_{\mathrm{ph}}$ at the substrate surface are plotted for two laser pulses with a pulse separation of $\Delta t=1 \mathrm{ps}$. Assuming a purely electronmediated energy transfer [11], the adsorbate temperatures $T_{\text {ads }}$ for an $\mathrm{H}$ and $\mathrm{D}$ saturation layer, respectively, result. In the lower section, the corresponding rates and their time integrals, the desorption yields, are displayed. (b) Illustration of the electronic processes after fs-laser excitation.

(within $\leq 100$ fs [18]) and subsequently cool down by coupling to the lattice characterized by its phonon temperature $T_{\mathrm{ph}}$. The distribution of the hot electrons is governed by $T_{\text {el }}$ through Fermi-Dirac statistics. As a consequence, an adsorbate derived affinity level above $E_{\mathrm{F}}$ may become partially occupied, and the system is transferred to another electronically excited potential. The gradient on this potential initiates nuclear motion during the short lifetime (typically $\leq 10$ fs [17]). Relaxation occurs by electron transfer back to the substrate, and thereby the system returns to the ground state potential. This electronically nonadiabatic process causes coupling between electronic and nuclear degrees of freedom [19] and is also denoted as electronic friction [11,20]. Its multiple repetition eventually enables the adsorbed species to take up enough energy to react and desorb from the surface (desorption induced by multiple electronic transitions [17]).

If the surface is saturated with hydrogen, but with a varying proportion of both isotopes $\mathrm{H}$ and $\mathrm{D}$, i.e., constant $(1 \times 1)$ coverage, the total yields of $\mathrm{H}_{2}, \mathrm{D}_{2}$, and $\mathrm{HD}$ coming off in the TDS experiment should follow second order rate equations of the forms $d / d t\left[\mathrm{H}_{2}\right]=k[\mathrm{H}]^{2}$, $d / d t\left[\mathrm{D}_{2}\right]=k[\mathrm{D}]^{2}$, and $d / d t[\mathrm{HD}]=2 k[\mathrm{H}][\mathrm{D}]$. These differential equations are coupled through the mass continu- ities $d / d t[\mathrm{H}]=-2 d / d t\left[\mathrm{H}_{2}\right]-d / d t[\mathrm{HD}]$ and $d / d t[\mathrm{D}]=$ $-2 d / d t\left[\mathrm{D}_{2}\right]-d / d t[\mathrm{HD}]$. It is assumed that there exists random mixing among both chemically identical adsorbates and both $\mathrm{H}_{2}$ and $\mathrm{D}_{2}$ desorb with the same (thermal) rate constant $k$ which is justified by the TDS data in Fig. 1(a). Indeed, the experimental TDS yields show excellent agreement with theory [solid lines in Fig. 4(a)] and demonstrate that thermal desorption can be rationalized in terms of a simple bimolecular reaction.

The first-shot yields resulting from fs-pulse excitation are shown in Fig. 4(b). In contrast to the TDS data, now $\leq 5 \%$ of the total coverage is released into the gas phase so that the data reflect the influence of the adsorbate composition on desorption from a fully covered surface to a good approximation [21]. The asymmetry in the laser-induced desorption yields is caused by the pronounced isotope effect between the pure adlayers already mentioned. Very intriguing discrepancies are found when one compares the experimental data with calculations [Fig. 5(a)] using the time-dependent rate constants $k\left[T_{\text {ads }}(t)\right]$ out of the friction model (see Fig. 3) in the rate equations introduced above [22]: the yields for $\mathrm{D}_{2}$ are appreciably higher and those for $\mathrm{H}_{2}$ lower than predicted. Because of the electronic nature of the energy transfer, the adsorbate temperatures $T_{\text {ads }}$ for pure $\mathrm{H}$ and $\mathrm{D}$ adlayers follow $T_{\mathrm{el}}$ with a certain delay $\Delta t_{\mathrm{H}}$ and $\Delta t_{\mathrm{D}}$ of 180 and $330 \mathrm{fs}$, respectively [Fig. 3(a)]. $T_{\text {ads }}(t)$ for $\mathrm{H}$ clearly reaches its maximum before the respective $T_{\text {ads }}$ for the heavier $\mathrm{D}$, reflecting the different coupling times as derived from the electronic friction model. Consequently, energy transfer into the adsorbate occurs twice as fast for $\mathrm{H}$ as for $\mathrm{D}$ and hence the more rapidly excited $\mathrm{H}$ atoms will start earlier to be displaced from their equilibrium positions thereby also affecting (neighboring) D atoms [Fig. 5(b)] [23]. We propose that the resulting changes in the electronic structure lead to the enhancement of the recombinative desorption of $\mathrm{D}_{2}$. One physical

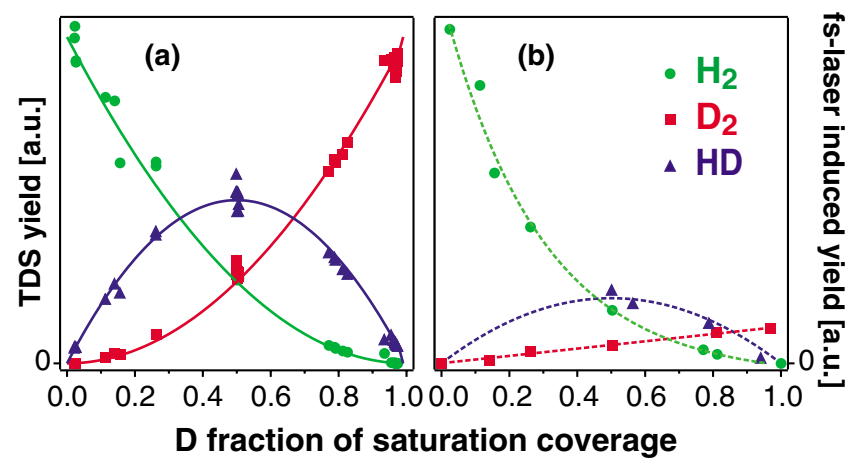

FIG. 4 (color). $\mathrm{H}_{2}, \mathrm{D}_{2}$, and $\mathrm{HD}$ desorption yield from a hydrogen-saturated $\mathrm{Ru}(0001)$ surface as a function of the $\mathrm{D}$ species concentration on the surface. (a) Thermal desorption by resistive heating. Solid lines are based on second order rate equations numerically integrated with the coupling continuity equations (see text). (b) Desorption induced by fs-laser excitation, reflecting the isotope effect of 10:1 between isotopically pure coverages. Dashed lines are guides to the eye. 


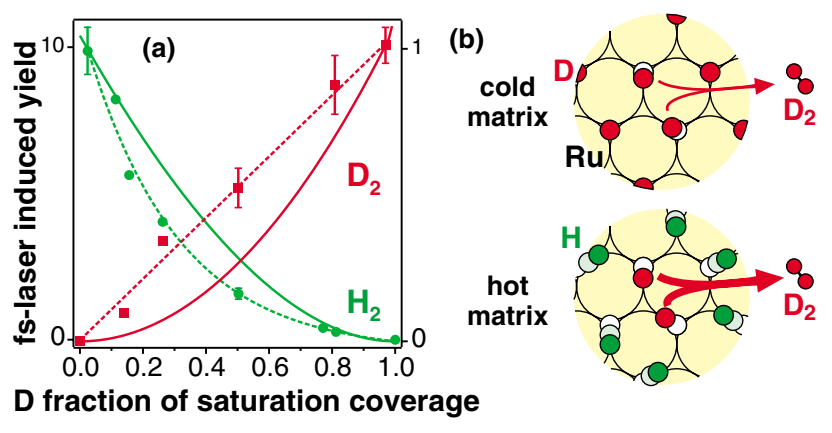

FIG. 5 (color). (a) Comparison of fs-laser induced $\mathrm{H}_{2}$ and $\mathrm{D}_{2}$ desorption yield with the outcome of second order rate equation calculations based on time-dependent rate constants (see text). (b) Top: In a cold matrix realized by a $\mathrm{D}$ surrounding, $\mathrm{D}_{2}$ recombination occurs between two excited reactants. Bottom: The more rapid excitation of $\mathrm{H}$ atoms creates a hot matrix, in which the recombination of $\mathrm{D}_{2}$ molecules is enhanced.

picture of the relevant microscopic motion would involve repulsive interactions in which $\mathrm{H}$ atoms give way to two $\mathrm{D}$ reactants. However, also nonlocal contributions to the promotion effect might play a role, as band structure changes induced even by low coverages in photoemission experiments from $\mathrm{H}$ on various metals indicate [24]. That the presence of neighboring $\mathrm{H}_{\mathrm{ad}}$ species obviously enhances the $\mathrm{D}_{2}$ recombination probability also applies to the $\mathrm{H}_{2}$ formation despite the apparent reduction of the $\mathrm{H}_{2}$ yield in the presence of a D surrounding [Fig. 5(a)]. Without the promotion effect for $\mathrm{H}$ atoms in an $\mathrm{H}$ surrounding, the isotope ratio between pure $\mathrm{H}$ and $\mathrm{D}$ coverages would have been smaller than the measured value of 10:1. Thus, the lighter isotope represents a dynamic promoter for the surface reaction of both $\mathrm{D}_{2}$ and $\mathrm{H}_{2}$ formation.

Our results imply that the rate constant $k$ for the recombination reaction from mixed adlayers needs to be reformulated: $k$ is no longer dependent only on a uniform adsorbate temperature $k=k\left(T_{\text {ads }}\right)$, but rather is a function of the H:D proportion, i.e., the isotope concentrations [H] and [D]. Consequently, $k$ might be rewritten as $k=$ $k\left(T_{\mathrm{ads}}^{\mathrm{H}}, T_{\mathrm{ads}}^{\mathrm{D}}\right)$. This concept also holds for thermally initiated desorption since under equilibrium conditions $T_{\mathrm{ads}}^{\mathrm{H}}=$ $T_{\text {ads }}^{\mathrm{D}}$, describing the adsorbate system by a uniform parameter. However, even in the thermal case, the site fluctuations of neighboring adatoms will cause desorption preferentially in moments when the surrounding has created a favorable energy landscape. A crucial test for the promotion effect would be ab initio calculations for $k$ solely accounting for both reactants compared to the experimental results reported here.

To summarize, our work on hydrogen recombination on Ru shows that femtosecond-laser excitation triggers an electron-mediated reaction pathway. The observed huge isotope effect is enhanced by a collective mechanism in which due to different energy coupling times the sur- rounding of the reactants dynamically changes their reactivity. Although observed in a fs-laser induced reaction, dynamical promotion might be a common feature in surface chemistry, but cannot be resolved in thermal surface reactions due to equilibrium conditions.

We thank A. P. Seitsonen and O. Pulci for helpful discussions and the DFG for financial support (Sfb 450).

[1] H. Eyring and M. Polanyi, Z. Phys. Chem. B 12, 279 (1931).

[2] M. Bonn et al., Science 285, 1042 (1999).

[3] B. Gergen et al., Science 294, 2521 (2001).

[4] The Chemical Physics of Solid Surfaces, edited by D. A. King and D. P. Woodruff (Elsevier, Amsterdam, 1993), Vol. 6.

[5] M. Lindroos et al., Surf. Sci. 180, 237 (1987).

[6] P. Feulner and D. Menzel, Surf. Sci. 154, 465 (1985).

[7] S. Funk et al., J. Chem. Phys. 112, 9888 (2000).

[8] P. Feulner and D. Menzel, J. Vac. Sci. Technol. 17, 662 (1980).

[9] W. H. Weinberg, Acc. Chem. Res. 29, 479 (1996).

[10] F. Budde et al., Phys. Rev. Lett. 66, 3024 (1991).

[11] M. Brandbyge et al., Phys. Rev. B 52, 6042 (1995).

[12] M. Bonn et al., Phys. Rev. B 61, 1101 (2000).

[13] S. I. Anisimov et al., Sov. Phys. JETP 39, 375 (1974).

[14] $E_{\mathrm{a}}$ in the desorption probability $E_{\mathrm{a}} \int d t\left[\tau_{\mathrm{el}} T_{\mathrm{ads}}(t)\right]^{-1} \times$ $\exp \left[-E_{\mathrm{a}} / k_{\mathrm{B}} T_{\mathrm{ads}}(t)\right]$ of Ref. [11] does not represent a genuine activation energy of an Arrhenius expression. However, $E_{\mathrm{a}}$ exceeds the desorption energy $E_{\text {des }}$ [see Fig. 3(b)] indicating the activation of electronically excited states.

[15] M. Yata and R. J. Madix, Surf. Sci. 328, 171 (1995).

[16] D. Menzel, Surf. Sci. 47, 370 (1975).

[17] J. A. Misewich et al., Phys. Rev. Lett. 68, 3737 (1992).

[18] M. Lisowski et al., Appl. Phys. A 78, 165 (2004).

[19] This electronically induced adsorbate motion represents the inverse of the vibrational relaxation at surfaces through electron-hole pair generation. In the case of the $\mathrm{H}_{2}$ recombination, the multidimensional reaction coordinate comprises the activation motions of both reactants.

[20] F. Budde et al., Surf. Sci. 283, 143 (1993).

[21] Note that surface diffusion is widely suppressed in coverages at and near saturation due to blocking of the next nearest neighbor sites and hence is negligible.

[22] Successfully describing the substrate-adsorbate energy transfer, the friction model assumes equilibrium in the adsorbate subsystem with temperature $T_{\text {ads }}$. Hence, the use of equilibrium rate equations seems reasonable.

[23] Any competition effects, in which a fast $\mathrm{H}_{2}$ desorption would reduce the reaction probability for HD formation and hence increase the $D_{2}$ yield, or changes in the electronic structure for the residual $\mathrm{D}$ share on the surface after an early departure of $\mathrm{H}_{2}$ can be ruled out due to the low laser fluences applied in the experiments.

[24] F. Greuter et al., Phys. Rev. B 33, 736 (1986). 